$\mathrm{DSF}-26 / 96$

hep-ph/9607226

\title{
Supernova Neutrino Energy Spectra and the MSW Effect
}

\author{
F. Buccella ${ }^{1}$, S. Esposito ${ }^{1}$, C. Gualdi ${ }^{2}$ and G. Miele ${ }^{1}$ \\ ${ }^{1}$ Dipartimento di Scienze Fisiche, Università di Napoli "Federico II", and INFN, Sezione \\ di Napoli, Mostra D'Oltremare Pad. 20, I-80125 Napoli, Italy. \\ 2 Dipartimento di Fisica, Università di Ferrara, and INFN, Sezione di Ferrara, Via del \\ Paradiso 12, I-44100 Ferrara, Italy.
}

\begin{abstract}
The distortions in the thermal energy spectra for neutrinos produced in a supernova when a resonant oscillation, MSW effect, occurs are determined. In order to show this effect for some relevant and representative examples of unified gauge models, we have chosen $S O(10)$, and $S U(5)_{S U S Y}, S O(10)_{S U S Y}$ with a particular scheme for fermion masses (DHR model). The analysis has been performed for two choices of neutrinos parameters, predicted by the above models, and capable to explain the solar neutrino problem. In both cases one observes a strong distortion in the electron neutrino energy spectrum. This effect, computed for a wide range of $S O(10)_{S U S Y}$ models has produced the same results of the previous supersymmetric ones.
\end{abstract}

PACS: $13.15 .+\mathrm{g} ; 14.60 . \mathrm{Pq}$

e-mail: sesposito@na.infn.it; miele@na.infn.it 


\section{Introduction}

The long-base neutrinos physics certainly represents a suitable arena to reveal the presence of fundamental effects, which due to their weakness would be at the moment out of the range of accelerators experiments. In particular, the explanation of the so-called solar neutrino problem [四] seems to suggest the presence of non-vanishing neutrino masses, and of a corresponding mixing à la Cabibbo-Kobayashi-Maskawa also in the leptonic sector of the standard model. Interestingly, since similar predictions are naturally obtained in the framework of unified gauge models for fundamental interactions, one can think to use the neutrino measurements to test the validity of such models [2, 3].

In general, if one assumes massive neutrinos, the flavour eigenstates do not coincide with the mass eigenstates, and thus the phenomenon of neutrino oscillations can arise [1]. In fact, neutrinos are produced by weak interactions in flavour eigenstates, whereas they propagates in space as energy eigenstates (mass eigenstates), and these two basis do not coincide. Hence, there is a non-vanishing probability that starting with neutrinos of given flavour, at a certain distance from the source one can detect a neutrino of different flavour. Unfortunately, for solar neutrinos, this simple oscillation mechanism (vacuum oscillations) is almost independent of the neutrinos energy, and thus it hardly reproduces the experimental defect in the $\nu_{e}$ emission, which seems to show a quite strong energy dependence [1]. A possible solution for the solar neutrino problem is then provided by the so-called resonant oscillation mechanism, the Mikheyev-Smirnov-Wolfenstein effect (MSW) [5].

According to the MSW mechanism, when neutrinos propagate in a medium rich of electrons, neutrino oscillations can receive a resonant enhancement due to the different interactions experienced by $\nu_{e}$ 's (through both charged and neutral current) with respect to $\nu_{\mu}$ 's and $\nu_{\tau}$ 's (through only neutral current) with the medium itself.

Let us consider for simplicity only two neutrino species, $\nu_{e}$ and $\nu_{x}$ with $x=\mu$ or $\tau$. In this case if we denote with $E$ the neutrino energy, the enhanced oscillation occurs only if the following resonance condition is satisfied [5]

$$
\frac{\Delta m^{2}}{2 E} \cos (2 \theta)=\sqrt{2} G_{F} N_{e}
$$

where $\Delta m^{2}$ is the squared masses difference between the two neutrinos, $\theta$ the vacuum mixing angle, $N_{e}$ the electron number density of the medium, and $G_{F}$ the Fermi coupling constant.

As one can see from (1), the resonance condition if applied to astrophysical frameworks, would allow to scan very low values of $\Delta m^{2}$ and $\theta$ by using quite low energy neutrinos, since very high densities can be achieved for large regions. One of these situations is certainly provided during a supernova explosion.

In this paper, we study how the MSW effect modifies the neutrinos energy spectra coming out from a supernova. The predictions are obtained for a wide range of unified gauge models. In particular, we have analyzed the $S O(10)$ predictions, as the most rele-

vant example of non supersymmetric unified model still compatible with the experimental 
bounds on proton lifetime [2, 3]. Furthermore, for the sake of completeness, we have also considered $S U(5)$ and $S O(10)$ supersymmetric unified models [6, 0, 8].

The paper is organized as follows. Section 2 is devoted to the study of the MSW effect for medium with varying density. In particular, the relevant quantities are defined and the expressions for neutrino spectra are obtained. In Section 3 we review the main predictions for the neutrino physics corresponding to the still viable unified gauge models, supersymmetric and not, and for these theories we compute the neutrinos spectra. Then in section 4 we give our conclusions and remarks.

\section{MSW effect in supernovæ}

In media with varying density the probability of neutrino conversion depends on the rate of such a variation. Within the adiabatic approximation [9], namely under the assumption that the medium density crossed by neutrinos changes very slowly, one predicts an high rate of conversion. Quantitatively, this approximation is valid when the adiabaticity parameter

$$
\gamma \equiv \frac{\Delta m^{2}}{2 E} \frac{\sin ^{2}(2 \theta)}{\cos (2 \theta)}\left|\frac{1}{N_{e}} \frac{d N_{e}}{d r}\right|_{r e s}^{-1},
$$

computed at the resonance point, is greater than 1 . In the opposite case, the survival probability would be of the order of unity, so that no appreciable conversion can be obtained. For neutrinos propagating in a given medium it is useful to introduce the adiabatic and non-adiabatic threshold energies

$$
\begin{aligned}
E_{A} & =\frac{\Delta m^{2} \cos (2 \theta)}{2 \sqrt{2} G_{F} N_{e}^{\text {prod }}}, \\
E_{N A} & =\frac{\pi \Delta m^{2} \sin ^{2}(2 \theta)}{4 \cos (2 \theta)}\left|\frac{1}{N_{e}} \frac{d N_{e}}{d r}\right|_{\text {res }}^{-1},
\end{aligned}
$$

where $N_{e}^{\text {prod }}$ is the electron density at the neutrinos production point回. The former indicates the transition between no matter effects and resonant conversion, described by the adiabatic approximation, while the latter represents the energy scale for the transition between the adiabatic and non-adiabatic regions.

The density range in a supernova is very large; it goes from $\sim 10^{-5} \mathrm{~g} / \mathrm{cm}^{3}$ in the external envelope up to $\sim 10^{15} \mathrm{~g} / \mathrm{cm}^{3}$ in the dense core. For our purposes, as a reasonable radial density profile one can use the following scaling law [9, 10]

$$
\rho \simeq \rho_{0}\left(\frac{R_{0}}{r}\right)^{3},
$$

with $\rho_{0} \simeq 3.5 \times 10^{10} \mathrm{~g} / \mathrm{cm}^{3}$, and $R_{0} \simeq 1.02 \times 10^{7} \mathrm{~cm}$. The quantity $R_{0}$ defines the so-called neutrinosphere, which represents the bounding surface of the region in which neutrinos of

\footnotetext{
${ }^{1}$ For simplicity, one can assume that this point essentially coincides with the neutrinosphere.
} 
a given flavour are in thermal equilibrium (eq. (5) is approximatively valid for $r \geq R_{0}$ ). Outside the neutrinosphere, the electron fraction number $Y_{e}$ can be assumed almost constant with respect to the radius, and thus the value $Y_{e}=0.42$ can be used. Since inside the neutrinosphere the resonance conditions are not satisfied, for a wide range of unified models, we can assume in first approximation that on this surface the neutrinos are emitted with an almost Fermi-Dirac distribution, and with the different flavors equally populated. However, flavour changing effects occurring inside the neutrinosphere have been proposed in literature [11], but in any case they would represent further corrections with respect to the bulk phenomenon we want to describe.

Consequently, from (四) it follows that neutrinos diffusing out the neutrinosphere experience two MSW resonances: the first resonance, between $\nu_{e}$ and $\nu_{\tau}$, at a density of about $10^{8} \mathrm{~g} / \mathrm{cm}^{3}$ and the second one, between $\nu_{e}$ and $\nu_{\mu}$, at about $10^{2} \mathrm{~g} / \mathrm{cm}^{3}$. These values refer to average neutrino energies $\bar{E}_{\nu_{e}} \simeq 10 \mathrm{MeV}, \bar{E}_{\nu_{\mu}, \nu_{\tau}} \simeq 20 \mathrm{MeV}$, and to the indicative values $\Delta m_{e \mu}^{2} \simeq 10^{-6} \mathrm{eV}^{2}, \Delta m_{e \tau}^{2} \simeq 25 \mathrm{eV}^{2}$ for the squared masses differences. The evolution of the different neutrino flavors can be simply summarized by the following diagrams

$$
\begin{array}{r}
\nu_{e}\left\{\begin{array}{l}
----->\nu_{\tau}--------->\nu_{\tau} \\
--------->\nu_{\mu}---->\nu_{\mu} \\
---------------->\nu_{e}
\end{array}\right. \\
\nu_{\mu}\left\{\begin{array}{r}
---------->\nu_{e}----->\nu_{e} \\
------------------>\nu_{\mu}
\end{array}\right. \\
\nu_{\tau}\left\{\begin{array}{r}
----->\nu_{e}---------->\nu_{e} \\
-->\nu_{\mu}----->\nu_{\mu}
\end{array}\right.
\end{array}
$$

Electron-neutrinos may oscillate into $\nu_{\tau}$ at the first resonance or into $\nu_{\mu}$ at the second resonance, whereas muon-neutrinos can oscillate only into $\nu_{e}$ at the second resonance. Finally, tau-neutrinos may oscillate into $\nu_{e}$ at the first resonance and the latter can further oscillate into $\nu_{\mu}$ at the second resonance.

To study the evolution of neutrino flavors it is crucial to know how these resonance regions are crossed, namely if adiabatically or non-adiabatically.

\subsection{The $\nu_{e}$ survival probability and spectra distortion}

In order to obtain the energy spectra of the emitted neutrinos from a supernova, let us focus our attention on neutrinos produced in the thermal phase, which diffuse out the neutrinosphere with a spectrum approximatively given by [10, 12]

$$
n_{\nu_{i}}(E) \simeq \frac{0.5546}{T_{i}^{3}} E^{2}\left[1+\exp \left(\frac{E}{T_{i}}\right)\right]^{-1},
$$

\footnotetext{
${ }^{2}$ Note that we assume neutrino masses negligible with respect to $T_{i}$.
} 
where the index $i$ denotes the particular neutrino specie. Since the production and scattering cross-sections for electron-neutrinos are larger than for the other flavors, $\nu_{e}$ are produced a bit more copiously with respect to the other ones. Thus, their neutrinosphere is larger than that for $\nu_{\mu}, \nu_{\tau}$. This implies that the temperature $T_{i}$ in (9) for the $\nu_{e}$ sphere is lower than the one of $\nu_{\mu}, \nu_{\tau}$. Furthermore, since $\nu_{\mu}$ and $\nu_{\tau}$ are produced and scatter on the surrounding matter only through neutral currents, they have identical spectra. Obviously, since $\nu_{e}, \nu_{\mu}, \nu_{\tau}$ and $\bar{\nu}_{e}, \bar{\nu}_{\mu}, \bar{\nu}_{\tau}$ are produced in pairs, the magnitude of neutrino and antineutrino fluxes are equal for each flavour. For the temperature of $\nu_{e}$ and $\nu_{\mu}, \nu_{\tau}$ neutrinosphere we adopt the typical values [10, [9]

$$
\begin{aligned}
& T_{e} \simeq 3 \mathrm{MeV}, \\
& T_{\mu}=T_{\tau} \simeq 6 \mathrm{MeV} .
\end{aligned}
$$

By indicating with $n_{\nu_{e}}^{0}$ and $n_{\nu_{x}}^{0} \equiv n_{\nu_{\mu}}^{0}=n_{\nu_{\tau}}^{0}$ the spectra of $\nu_{e}$ and $\nu_{\mu}, \nu_{\tau}$ at the relative neutrinospheres, according to the oscillation scheme previously outlined, we have that the spectra of the different neutrino flavors emerging from a supernova are

$$
\begin{aligned}
& n_{\nu_{e}}=P\left(\nu_{e} \rightarrow \nu_{e}\right) n_{\nu_{e}}^{0}+\left[P\left(\nu_{\mu} \rightarrow \nu_{e}\right)+P\left(\nu_{\tau} \rightarrow \nu_{e}\right)\right] n_{\nu_{x}}^{0}, \\
& n_{\nu_{\mu}}=P\left(\nu_{e} \rightarrow \nu_{\mu}\right) n_{\nu_{e}}^{0}+\left[P\left(\nu_{\mu} \rightarrow \nu_{\mu}\right)+P\left(\nu_{\tau} \rightarrow \nu_{e} \rightarrow \nu_{\mu}\right)\right] n_{\nu_{x}}^{0}, \\
& n_{\nu_{\tau}}=P\left(\nu_{e} \rightarrow \nu_{\tau}\right) n_{\nu_{e}}^{0}+P\left(\nu_{\tau} \rightarrow \nu_{\tau}\right) n_{\nu_{x}}^{0},
\end{aligned}
$$

where $P\left(\nu_{\alpha} \rightarrow \nu_{\beta}\right)$ is the probability for the transition $\nu_{\alpha} \rightarrow \nu_{\beta}$. Note that, from the experimental point of view, due to the relative low energy of the neutrinos considered $(0 \div 50 \mathrm{MeV})$, one cannot distinguish between $\nu_{\mu}$ and $\nu_{\tau}$, thus the only relevant quantities are $n_{\nu_{e}}$ and $n_{\nu_{\mu}}+n_{\nu_{\tau}}$. Consequently, by using the unitarity conditions on the transition probabilities (see diagrams (6)-(8)) we obtain

$$
\begin{aligned}
n_{\nu_{e}} & =P\left(\nu_{e} \rightarrow \nu_{e}\right) n_{\nu_{e}}^{0}+\left[1-P\left(\nu_{e} \rightarrow \nu_{e}\right)\right] n_{\nu_{x}}^{0}, \\
n_{\nu_{\mu}}+n_{\nu_{\tau}} & =\left[1-P\left(\nu_{e} \rightarrow \nu_{e}\right)\right] n_{\nu_{e}}^{0}+\left[1+P\left(\nu_{e} \rightarrow \nu_{e}\right)\right] n_{\nu_{x}}^{0}
\end{aligned}
$$

From the above equations it is evident that the only relevant quantity to be computed from the MSW theory is the probability that $\nu_{e}$ survives to both $\nu_{e} \rightarrow \nu_{\tau}$ and $\nu_{e} \rightarrow \nu_{\mu}$ resonances. Since for a wide range of unified models the two resonances are well separated we can write

$$
P\left(\nu_{e} \rightarrow \nu_{e}\right) \simeq P_{e \tau}\left(\nu_{e} \rightarrow \nu_{e}\right) P_{e \mu}\left(\nu_{e} \rightarrow \nu_{e}\right),
$$

where $P_{e \tau}\left(\nu_{e} \rightarrow \nu_{e}\right)$ and $P_{e \mu}\left(\nu_{e} \rightarrow \nu_{e}\right)$ are the survival probabilities for $\nu_{e}$ 's to the first and second resonance. These quantities can be calculated by using a simple two flavour approach. According to this we have [9]

$$
P_{e \mu}\left(\nu_{e} \rightarrow \nu_{e}\right)=\frac{1}{2}+\left(\frac{1}{2}-P_{e \mu}^{c}\right) \cos \left(2 \theta_{e \mu}\right) \cos \left(2 \theta_{m}^{e \mu}\right)
$$


and an analogous form for $P_{e \tau}\left(\nu_{e} \rightarrow \nu_{e}\right)$. Here $\theta_{m}$ denotes the effective mixing angle in matter calculated at the neutrinosphere. It is given by

$$
\cos \left(2 \theta_{m}\right)= \begin{cases}\operatorname{sign}\left(E_{A}-E\right)\left[1+\tan ^{2}(2 \theta)\left(\frac{E_{A}}{E_{A}-E}\right)^{2}\right]^{-1 / 2} & E \neq E_{A} \\ 0 & E=E_{A}\end{cases}
$$

where $P^{c}$ is the probability of level crossing at the resonance. For the density scaling law (5), it has the expression

$$
P^{c}=\exp \left[-\left(\frac{E_{N A}^{*}}{E}\right)^{\frac{2}{3}} F(\theta)\right]
$$

where the function $F(\theta)$ is approximatively given by

$$
F(\theta) \simeq\left(1-\tan ^{2}(\theta)\right)\left\{1+\frac{1}{3}\left[\log \left(1-\tan ^{2}(\theta)\right)+1-\frac{1+\tan ^{2}(\theta)}{\tan ^{2}(\theta)} \log \left(1+\tan ^{2}(\theta)\right)\right]\right\}
$$

and $E_{N A}^{*}$ is the effective non-adiabatic threshold energy for density scaling as $1 / r^{3}$

$$
\left(\frac{E_{N A}^{*}}{1 M e V}\right)^{\frac{2}{3}} \simeq 2.4 \times 10^{6}\left(\frac{\Delta m^{2}}{1 e V^{2}}\right)^{\frac{2}{3}} \frac{\sin ^{2}(2 \theta)}{[\cos (2 \theta)]^{\frac{4}{3}}} Y_{e}^{\frac{1}{3}}
$$

\section{Supernova neutrino spectra for unified gauge mod- els}

Let us apply the previous results to some relevant example of unified gauge models. In particular, we will consider the unified gauge model based on $S O(10)$ [2, 3] which among the non supersymmetric GUT theories, represents the most appealing unification scheme. Furthermore, we will extend our analysis to the SUSY version of $S U(5)$ and $S O(10)$ recently extensively discussed in literature [6, 7, 8].

\section{1 $S O(10)$ GUT models and neutrino predictions}

As already noted in the previous section, the MSW effect strongly depends on the neutrino mixing angle $\theta$ and on $\Delta m^{2}$. For $\nu_{e} \rightarrow \nu_{\mu}$ transitions, however, one can restrict the values of these quantities by requiring that MSW explains the solar neutrino problem [1]. This analysis, performed by one of the present authors [13] has given the following results

$$
\begin{aligned}
\Delta m_{e \mu}^{2} & \simeq 6 \times 10^{-6} e V^{2}, \\
\sin ^{2}\left(2 \theta_{e \mu}\right) & \simeq 7 \times 10^{-3} .
\end{aligned}
$$


A value for the mass of $\nu_{\mu}$ of the order of the square root of the r.h.s. of Eq. (23) (and a value for the mass of $\nu_{e}$ several order of magnitude smaller) is found in $S O(10)$ models with intermediate symmetry $S U(4)_{P S}\left(\right.$ or $\left.S U(3)_{C} \otimes U(1)_{B-L}\right) \otimes S U(2)_{L} \otimes S U(2)_{R}$. In general, a mass for $\nu_{\tau}$ about $m_{t}^{2} / m_{c}^{2}$ larger than the one of $\nu_{\mu}$ is expected [14]. This implies, provided $m_{\nu_{e}}<<m_{\nu_{\mu}}$,

$$
m_{\nu_{\tau}} \approx 35 \mathrm{eV}
$$

larger than $\sim 5 \mathrm{eV}$ which is the value found if one identifies $\nu_{\tau}$ as the hot component of dark matter [15]. However, the value given by Eq. (25) is obtained by assuming equal Majorana masses for $\nu_{\mu R}$ and $\nu_{\tau R}$. Thus, the factor 7 of discrepancy may be just ascribed to this ansatz. For this reason one can safely take for $m_{\nu_{\tau}}$ the value $5 \mathrm{eV}$.

By applying Eq. (3) this value corresponds to an adiabatic threshold for the $\nu_{e} \Leftrightarrow \nu_{\tau}$ transition in the matter of about $10^{-2} \mathrm{MeV}$ (since we expect small mixing angle we take $\left.\cos \left(2 \theta_{e \tau}\right) \simeq 1\right)$. The efficiency in the conversion $\nu_{e} \Leftrightarrow \nu_{\tau}$ depends on the value of the mixing angle between $\nu_{e}$ and $\nu_{\tau}$. To be more precise, it can be large if the threshold of the non adiabatic region is larger than the adiabatic threshold, or small in the opposite case, if the adiabatic region vanishes.

Let us denote with $\tilde{\theta}_{e \tau}$ the value of the mixing angle for which the two thresholds (3) and (22) coincide. With the density scaling given by (5) we get

$$
\sin ^{2}\left(2 \tilde{\theta}_{e \tau}\right)=3.25 \times 10^{-9} .
$$

Therefore, for $\tilde{\theta}_{e \tau}>\theta_{e \tau}$, the MSW conversion $\nu_{e} \Leftrightarrow \nu_{\tau}$ will be important, otherwise it will be small. Then in the following analysis we will consider the two values $\sin ^{2}\left(2 \theta_{e \tau}\right)=$ $3.2510^{-8}$ and $3.25 \times 10^{-10}$, representatives of the two widely different regimes discussed above.

By using the above values for neutrino parameters we can apply the MSW theory to obtain the spectra of emitted neutrinos from a supernova. In particular, Figure 1 shows the adiabaticity parameter $\gamma$ for $\nu_{e} \rightarrow \nu_{\mu}$ (solid line) and $\nu_{e} \rightarrow \nu_{\tau}$ transitions (dashed line), respectively, versus the neutrino energy. Note that, the dashed line corresponds to the larger value for $\sin ^{2}\left(2 \theta_{e \tau}\right)$, since to the other choice just would correspond a line two order of magnitude lower.

The plots show clearly that the $\nu_{e} \rightarrow \nu_{\tau}$ resonance is crossed much less adiabatically than $\nu_{e} \rightarrow \nu_{\mu}$ if values for $\sin ^{2}\left(2 \theta_{e \tau}\right)<<3.25 \times 10^{-9}$ are considered. Hence in these cases, the transition $\nu_{e} \rightarrow \nu_{\mu}$ is expected to be more effective than $\nu_{e} \rightarrow \nu_{\tau}$.

In Figures 2 and 3, 4 the $\nu_{e}$ survival probabilities, corresponding to the resonant transitions $\nu_{e} \rightarrow \nu_{\mu}$ and $\nu_{e} \rightarrow \nu_{\tau}$ respectively, are plotted versus the neutrino energy. From Figure 3 the extreme non-adiabatic nature of the $\nu_{e} \rightarrow \nu_{\tau}$ transition for $\sin ^{2}\left(2 \theta_{e \tau}\right) \simeq$ $3.25 \times 10^{-10}$ is clear.

In terms of the survival probability one can calculate the modifications of neutrino energy spectra as induced by MSW oscillations. In Figures 5 and 6 are plotted $n_{\nu_{e}}^{0}$, $n_{\nu_{e}}$ and $n_{\nu_{\mu}}^{0}+n_{\nu_{\tau}}^{0}, n_{\nu_{\mu}}+n_{\nu_{\tau}}$ respectively as given by equations (15), (16). In particular, the dashed and dotted lines represents the $S O(10)$ predictions for the values $\sin ^{2}\left(2 \theta_{e \tau}\right)=$ $3.25 \times 10^{-10}$ and $3.25 \times 10^{-8}$, respectively. 
As one can see from these Figures, the $\nu_{e}$ spectrum becomes harder toward higher energies (more easily detectable) while $\nu_{\mu}, \nu_{\tau}$ emerge from a supernova with lower energies. The integral flux of $\nu_{e}$ is also partially reduced of about $16 \%$.

However, the possible values of the neutrino mixing angles in $S O(10)$ models with fermion couplings to a $\underline{126}$ and a complex $\underline{10}$, as it is the case of the models discussed in Ref. [2], has been widely discussed by Babu and Mohapatra [3]. They conclude that it is possible to get the $\nu_{e} \Leftrightarrow \nu_{\mu}$ MSW mechanism advocated to explain the solar neutrino experiments with $\sin ^{2}\left(2 \theta_{e \tau}\right) \approx 10^{-2}$, much larger than the r.h.s. of Eq. (26). In this case the $\nu_{e} \Leftrightarrow \nu_{\tau}$ transition in supernovae is extremely efficient and thus the corresponding $P_{e \tau}\left(\nu_{e} \rightarrow \nu_{e}\right)$ in Eq. (17) is almost vanishing. Thus, the expressions of the neutrino spectra, as can be seen from (12)-(14), take the simple form

$$
\begin{aligned}
& n_{\nu_{e}}=n_{\nu_{x}}^{0}, \\
& n_{\nu_{\mu}}=n_{\nu_{x}}^{0} \\
& n_{\nu_{\tau}}=n_{\nu_{e}}^{0}
\end{aligned},
$$

It is interesting to observe that in this case the $\nu_{\tau}$ and $\nu_{e}$ distributions just interchange, whereas the $\nu_{\mu}$ one remains unmodified. This result can be easily understood by observing that for such an efficient $\nu_{e} \Leftrightarrow \nu_{\tau}$ transition, all the final $\nu_{e}$ come either from the former $\nu_{\tau}$, not subsequently transformed in $\nu_{\mu}$, or from the initial $\nu_{\mu}$ just transformed in $\nu_{e}$. Thus, since the initial distributions of $\nu_{\mu}$ and $\nu_{\tau}$ are assumed equal, this yields to the result (27)-(29).

\section{2 $S U(5)_{S U S Y}$ and $S O(10)_{S U S Y}$ unified models and neutrino pa- rameters}

As an example of a supersymmetric grand unified model, we consider the scheme discussed by Dimopoulos, Hall and Raby (DHR) [6] which is based on the Georgi-Jarlskog texture [16] for fermion mass matrices at the GUT scale. A peculiarity of the DHR model is that it can be realized both in $S U(5)_{S U S Y}$ and in $S O(10)_{S U S Y}$ GUT models (obviously, the Higgs multiplets in the two cases are different). The predictions for the neutrino mass ratios of this model are

$$
\begin{aligned}
& \frac{m_{\nu_{\mu}}}{m_{\nu_{e}}}=9 k^{4} \frac{m_{c}}{m_{t}}, \\
& \frac{m_{\nu_{\tau}}}{m_{\nu_{\mu}}}=\frac{1}{3 k^{2}}\left(\eta_{3} V_{c b}^{q}\right)^{-2},
\end{aligned}
$$

where $k=1$ or $-1 / 3$ (depending on particular choices in the DHR framework, see [6]), and $\eta_{3}=\eta_{c}\left(V_{c b}^{q}\right)^{2} m_{t} / m_{c}, \eta_{c}$ being the QCD renormalization enhancement factor of the charm mass between $m_{t}$ and $m_{c}$. The three leptonic mixing angles $\left(\theta_{1}^{\prime}, \theta_{2}^{\prime}, \theta_{3}^{\prime}\right)$ are expressed in terms of the three quark mixing angles $\left(\theta_{1}, \theta_{2}, \theta_{3}\right)$ by

$$
\sin \left(\theta_{1}^{\prime}\right)=-\frac{1}{3} \sin \left(\theta_{1}\right),
$$




$$
\begin{aligned}
& \sin \left(\theta_{2}^{\prime}\right)=\frac{1}{3 k^{2}} \sin \left(\theta_{2}\right), \\
& \sin \left(\theta_{3}^{\prime}\right)=2 k \eta_{3} \sin \left(\theta_{3}\right) \text {. }
\end{aligned}
$$

In particular,

$$
\begin{aligned}
& \theta_{e \mu} \simeq \sqrt{\frac{m_{e}}{m_{\mu}}+\frac{m_{\nu_{e}}}{m_{\nu_{\mu}}}-2 \sqrt{\frac{m_{e}}{m_{\mu}} \frac{m_{\nu_{e}}}{m_{\nu_{\mu}}}} \cos (\phi)} \\
& \theta_{e \tau} \simeq \sin \left(\theta_{1}^{\prime}\right) \sin \left(\theta_{3}^{\prime}\right)
\end{aligned}
$$

where $\phi$ is the phase of the leptonic CKM matrix.

Inserting in the above formulas the known experimental parameters, one obtains [6]

$$
\begin{aligned}
& \frac{m_{\nu_{\mu}}}{m_{\nu_{e}}}=\left\{\begin{array}{ll}
3100 \pm 1000 & \text { I } \\
38 \pm 12 & \text { II }
\end{array},\right. \\
& \frac{m_{\nu_{\tau}}}{m_{\nu_{\mu}}}=\left\{\begin{array}{ll}
208 \pm 42 & \text { I } \\
1870 \pm 370 & \text { II }
\end{array}\right. \text {, } \\
& \sin ^{2}\left(2 \theta_{e \mu}\right)=\left\{\begin{array}{ll}
(1.7 \pm 0.2) 10^{-2} & \text { I } \\
(9.0 \pm 4.3) 10^{-2} & \text { II }
\end{array}\right. \text {, } \\
& \sin ^{2}\left(2 \theta_{e \tau}\right)=\left\{\begin{array}{ll}
(1.3 \pm 0.3) 10^{-6} & \text { I } \\
(1.4 \pm 0.3) 10^{-7} & \text { II }
\end{array},\right.
\end{aligned}
$$

where I and II refer to the different values of $k$.

In this framework, as done in the previous section, one can fix the $\nu_{e} \rightarrow \nu_{\mu}$ mixing angle and squared masses difference to those values capable to explain the solar neutrino problem via the MSW mechanism $\left(\sin ^{2}\left(2 \theta_{e \mu}\right)=6 \times 10^{-4} \div 2 \times 10^{-2}, \Delta m_{e \mu}^{2}=(4 \div 9) 10^{-6} \mathrm{eV}^{2}\right.$ , see [1]). This requirement selects the model I with the following squared masses differences and mixing angles

$$
\begin{aligned}
\Delta m_{e \mu}^{2} & \simeq 6 \times 10^{-6} e V^{2} \\
\Delta m_{e \tau}^{2} & \simeq 0.27 e V^{2} \\
\sin ^{2}\left(2 \theta_{e \mu}\right) & \simeq 1.7 \times 10^{-2} \\
\sin ^{2}\left(2 \theta_{e \tau}\right) & \simeq 1.3 \times 10^{-6}
\end{aligned}
$$

In Figures 5 and 6 the predictions for the neutrino spectra for the DHR model, which is compatible with both $S U(5)_{S U S Y}$ and $S O(10)_{S U S Y}$, are shown (dashed-dotted lines).

As a possible alternative scenario, Lee and Mohapatra [8] have also considered the possibility of SUSY $S O(10)$, directly broken at a scale $\sim 10^{16} \mathrm{GeV}$ to $S U(3) \otimes S U(2) \otimes$ $U(1)$. In this scheme one expects Majorana masses for the $\nu_{R}$ 's at the highest scale and it is the $\nu_{\tau}$ the neutrino expected to have a mass around $10^{-3} \mathrm{eV}$. In that case the mass of $\nu_{\mu}$ is too small to cause a $\nu_{e} \Leftrightarrow \nu_{\mu}$ MSW conversion in an experimentally interesting range of neutrino energies. In this case, in supernova one should have only the conversion $\nu_{e} \Leftrightarrow \nu_{\tau}$, responsible for the solution of the solar neutrino problem. The situation would be 
very similar, as long as the distortions of neutrino spectrum are concerned, to a situation, where the MSW $\nu_{e} \Leftrightarrow \nu_{\mu}$ conversion is responsible for the solution of the solar neutrino problem and the mixing angle $\theta_{e \tau}$ is smaller than $\tilde{\theta}_{e \tau}$.

\section{Conclusions}

In this paper we have analyzed the distortions induced on the thermal energy spectra of $\nu_{e}$ and $\nu_{\mu}+\nu_{\tau}$ emitted from a supernova due to MSW effect. In order to study this phenomenon for some relevant examples of unification scenarios, we consider as possible choices of the neutrinos parameters, namely masses and mixings, the ones corresponding to the $S O(10)$ gauge group [2, 3], and the $S U(5)_{S U S Y}, S O(10)_{S U S Y}$ unified models in the scheme DHR [6], respectively. Other different $S O(10)_{S U S Y}$ models have been also considered [7], and interestingly, as far as the neutrino spectra predictions are concerned, it yields to results almost equal to the previous supersymmetric ones. Note that, in all cases $\Delta m_{e \mu}^{2}$ and $\sin ^{2}\left(2 \theta_{e \mu}\right)$ have been chosen to be compatible with the explanation of the solar neutrino problem [1].

According to the typical values for the density distribution in a supernova, out of the neutrinosphere, the condition of resonance is satisfied for the two flavour transitions $\nu_{e} \rightarrow$ $\nu_{\mu}$ and $\nu_{e} \rightarrow \nu_{\tau}$, respectively. By computing the corresponding adiabaticity parameter $\gamma$, one observes that, at least for $S O(10)$ GUT theories, the resonance $\nu_{e} \rightarrow \nu_{\tau}$ is crossed less adiabatically than $\nu_{e} \rightarrow \nu_{\mu}$ if one takes values $\sin ^{2}\left(2 \theta_{e \tau}\right)<<3.25 \times 10^{-9}$, and thus the latter results the most efficient. The survival probabilities for the electron-neutrinos corresponding to the above resonances are then obtained.

Finally, the distortion of the energy spectra are obtained for the $\nu_{e}$ and $\nu_{\mu}+\nu_{\tau}$, emitted from the supernova neutrinospheres in pure thermal equilibrium distributions. Remarkably, for both models $S O(10)$ and $S U(5)_{S U S Y}, S O(10)_{S U S Y}$ in DHR scheme, the distortion in the $\nu_{e}$ distribution is quite relevant, and moves neutrinos from the low energy region of the spectrum to the high energy sector. As a result of this analysis, one observes that the reduction of $\nu_{e}$ flux for $S O(10)$ and for the DHR model is of the order $16 \%$. Thus it is essentially model independent. This feature can be easily understood by observing that the relevant (since almost adiabatic) neutrino transition is $\nu_{e} \rightarrow \nu_{\mu}$, whose parameters are fixed from the MSW explanation for the solar neutrino problem.

The distortion in the $\nu_{e}$ distribution becomes extreme when the choice of neutrino parameteres discussed in the papers of Babu and Mohapatra [3] is taken. In this case, in fact, since the survival probability $P_{e \tau}\left(\nu_{e} \rightarrow \nu_{e}\right)$ is almost vanishing, we have the exact interchange of $\nu_{e}$ and $\nu_{\tau}$ distributions. 


\section{References}

[1] R. Davis Jr., Proc. of the 23rd ICRC, Calgary, Canada (1993), Prog. in Nucl. and Part. Phys. 32 (1994);

K. Hirata et al, Phys. Rev. D44 (1991) 2241;

A. Abazov et al, Phys. Rev. Lett. 67 (1991) 3332;

P. Anselmann et al., Phys. Lett. B327 (1994) 377.

[2] F. Buccella and L. Rosa, Zeit. für Phys. C36 (1987) 425;

M.F. Abud, F. Buccella, L. Rosa and A. Sciarrino, Zeit. für Phys. C44 (1989) 589;

R.N. Mohapatra and M.K. Parida, Phys. Rev. D47 (1993) 264;

F. Acampora, G. Amelino-Camelia, F. Buccella, O. Pisanti, L. Rosa, and T. Tuzi, Nuovo Cim. A108 (1995) 375;

G. Amelino-Camelia, O. Pisanti and L. Rosa, Nucl. Phys. (Proc. Suppl.) B43 (1995) 86.

[3] K.S. Babu and R.N. Mohapatra, Phys. Rev. Lett. 70 (1993) 2845;

Dae-Gyu Lee and R.N. Mohapatra, Phys. Lett. B329 (1994) 463.

[4] B. Pontecorvo, Sov. Phys. JETP V (1957) 1297;

B. Pontecorvo, Sov. Phys. JETP VI (1958) 429;

B. Pontecorvo, Sov. Phys. JETP VII (1958) 171.

[5] L. Wolfenstein, Phys. Rev. D17 (1978) 236;

S.P. Mikheyev and A.Yu. Smirnov, Nuovo Cim. C9 (1986) 17; Sov. J. Nucl. Phys. 42 (1986) 913; Sov. Phys. Usp. 30 (1987) 759.

[6] S. Dimopoulos, L. S. Hall and S. Raby, Phys. Rev. D47 (1993) 3697.

[7] Y. Achiman and T. Greiner, Nucl. Phys. B443 (1995) 3.

[8] Dae-Gyu Lee and R.N. Mohapatra, Phys. Rev. D51 (1995) 1353.

[9] T.K. Kuo and J. Pantaleone, Rev. Mod. Phys. 61 (1989) 937.

[10] T. Janka and W. Hillebrandt, Astron. Astroph. Suppl. 78 (1989) 375.

[11] J. C. D'Olivo and J. F. Nieves, Int. J. Mod. Phys. A11 (1996) 141.

[12] W.C. Haxton, Phys. Rev. D36 (1987) 2283.

[13] S. Esposito, Mod. Phys. Lett. A8 (1993) 1557.

[14] T. Yanagida, in Proc. of the Workshop on Unified Theory and Barion Number of the Universe, (KEK, Japan), 1979;

M. Gell-Mann, P. Ramond and R. Slansky, in Supergravity, P. Van Nieuwenhuizen and D.Z.Freedman eds., 1979, Amsterdam, North Holland, 315. 
[15] G.B. Gelmini, hep-ph/9606409;

Q. Shafi and F. Stecker, Phys. Rev. Lett. 53 (1984) 1292;

C.P. Ma and E. Bertscihinger, Ap. J. 429 (1994) 22, and Ap. J. 434 (1994) L5;

J. Primak et al., Phys. Rev. Lett. 74 (1995) 2160.

[16] H. Georgi and C. Jarlskog, Phys. Lett. B86 (1979) 297. 
Fig. 1

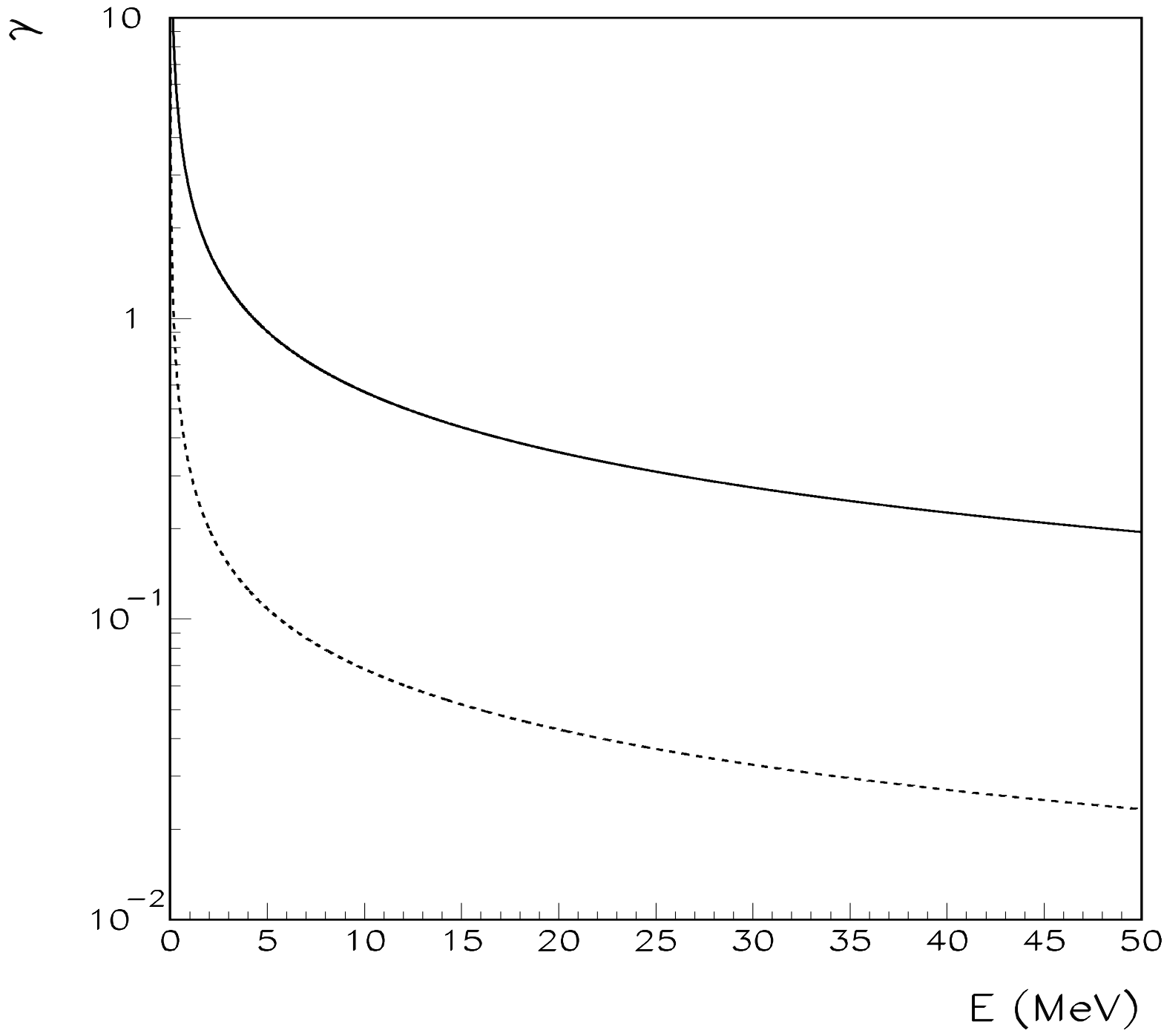

Figure 1: The adiabaticity parameter $\gamma$ for $\nu_{e} \rightarrow \nu_{\mu}$ (solid line) and for $\nu_{e} \rightarrow \nu_{\tau}$ transitions (dashed line) versus the neutrino energy is reported, for the $S O(10)$ unified model [3]. Note that for the $S O(10)$ case the line corresponds to $\sin ^{2}\left(2 \theta_{e \tau}\right)=3.2510^{-8}$. 
Fig. 2

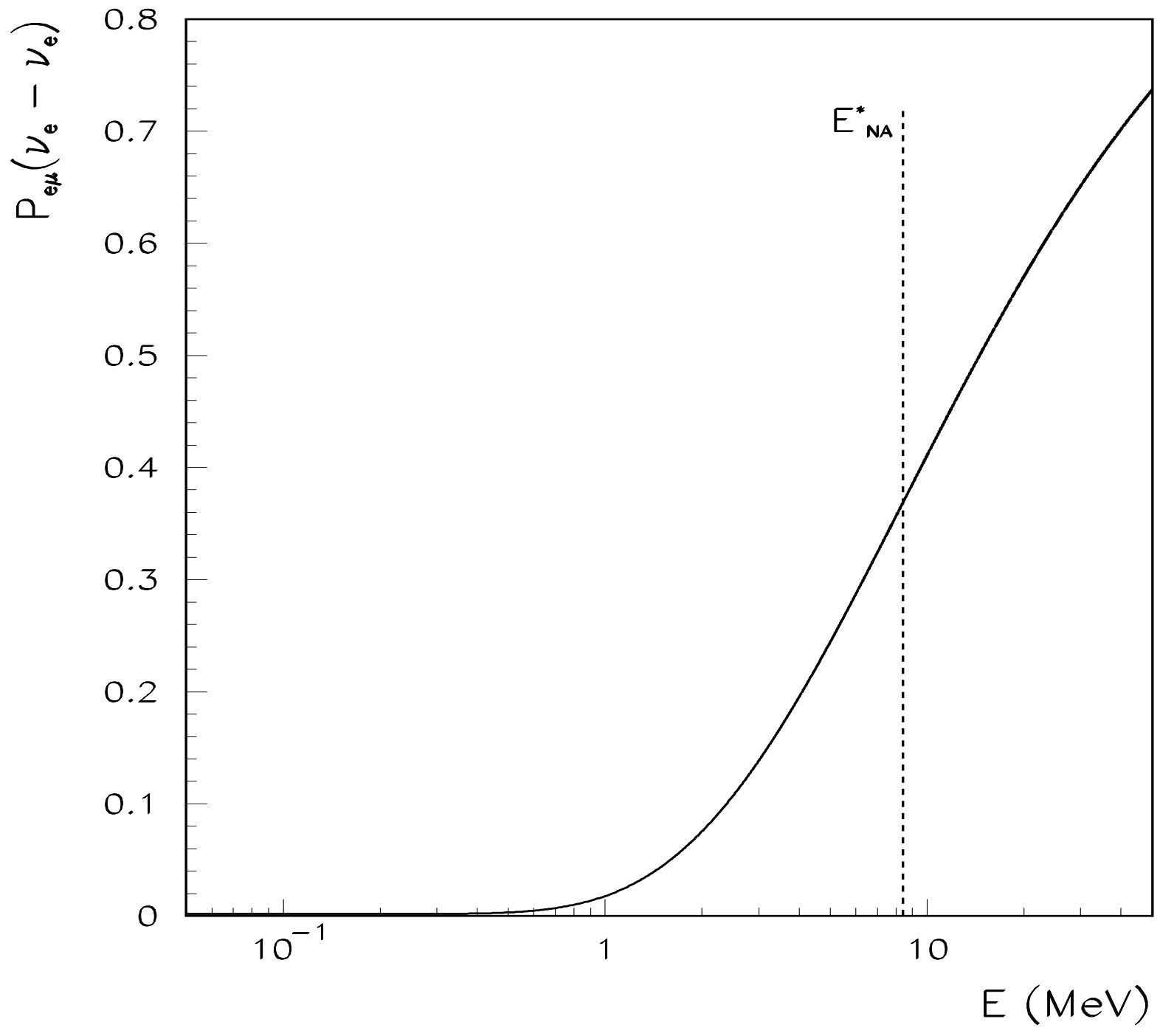

Figure 2: The survival probability $P_{e \mu}\left(\nu_{e} \rightarrow \nu_{e}\right)$ as function of the neutrino energy is shown for the $S O(10)$ [2]. 
Fig. 3

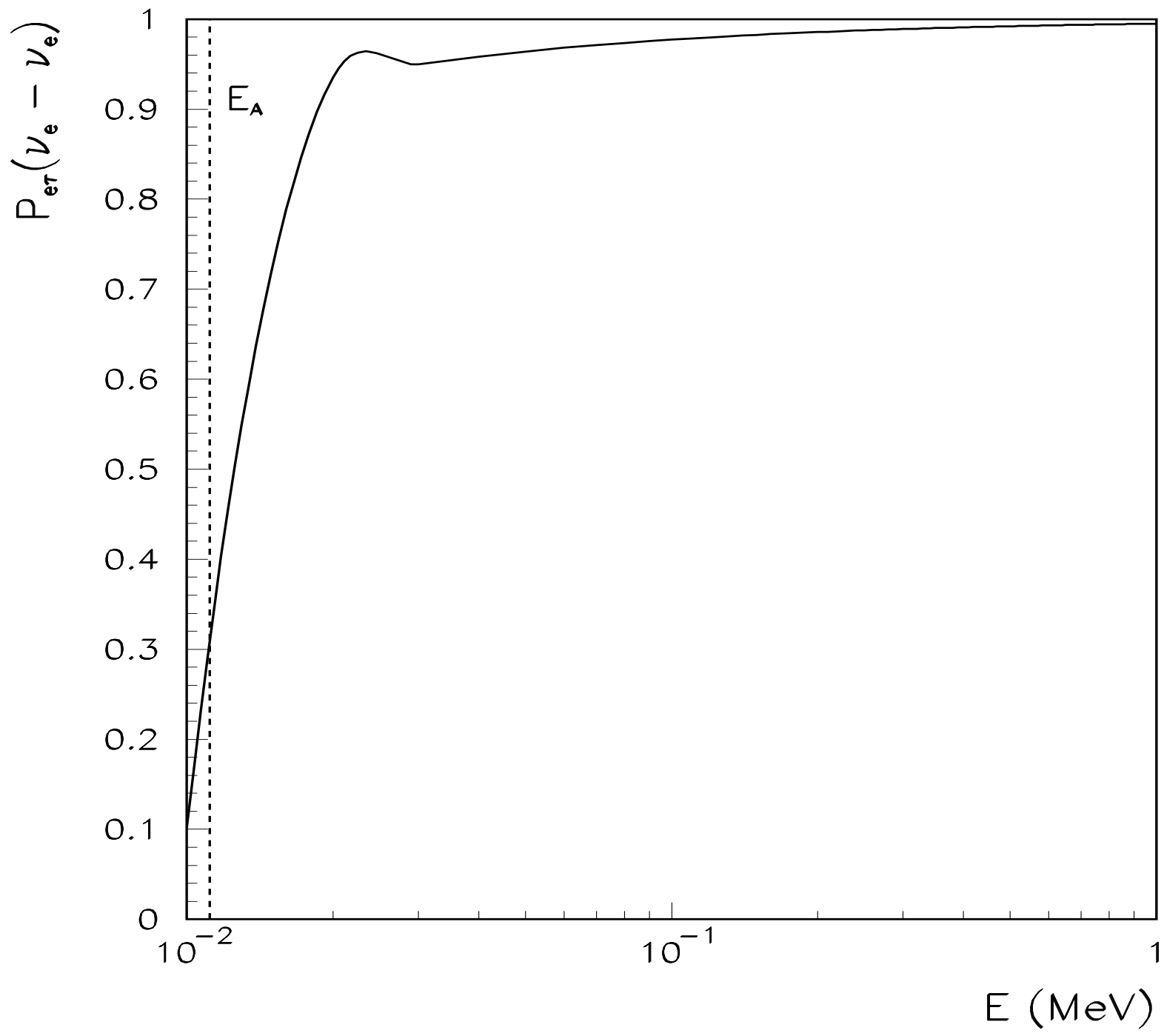

Figure 3: The survival probability $P_{e \tau}\left(\nu_{e} \rightarrow \nu_{e}\right)$ as function of the neutrino energy is shown for the $S O(10)$ [2], for $\sin ^{2}\left(2 \theta_{e \tau}\right)=3.25 \times 10^{-10}$. Note that, the corresponding value of $E_{N A}^{*}$, not reported in the figure, results to be much lower than $E_{A}$. 
Fig. 4

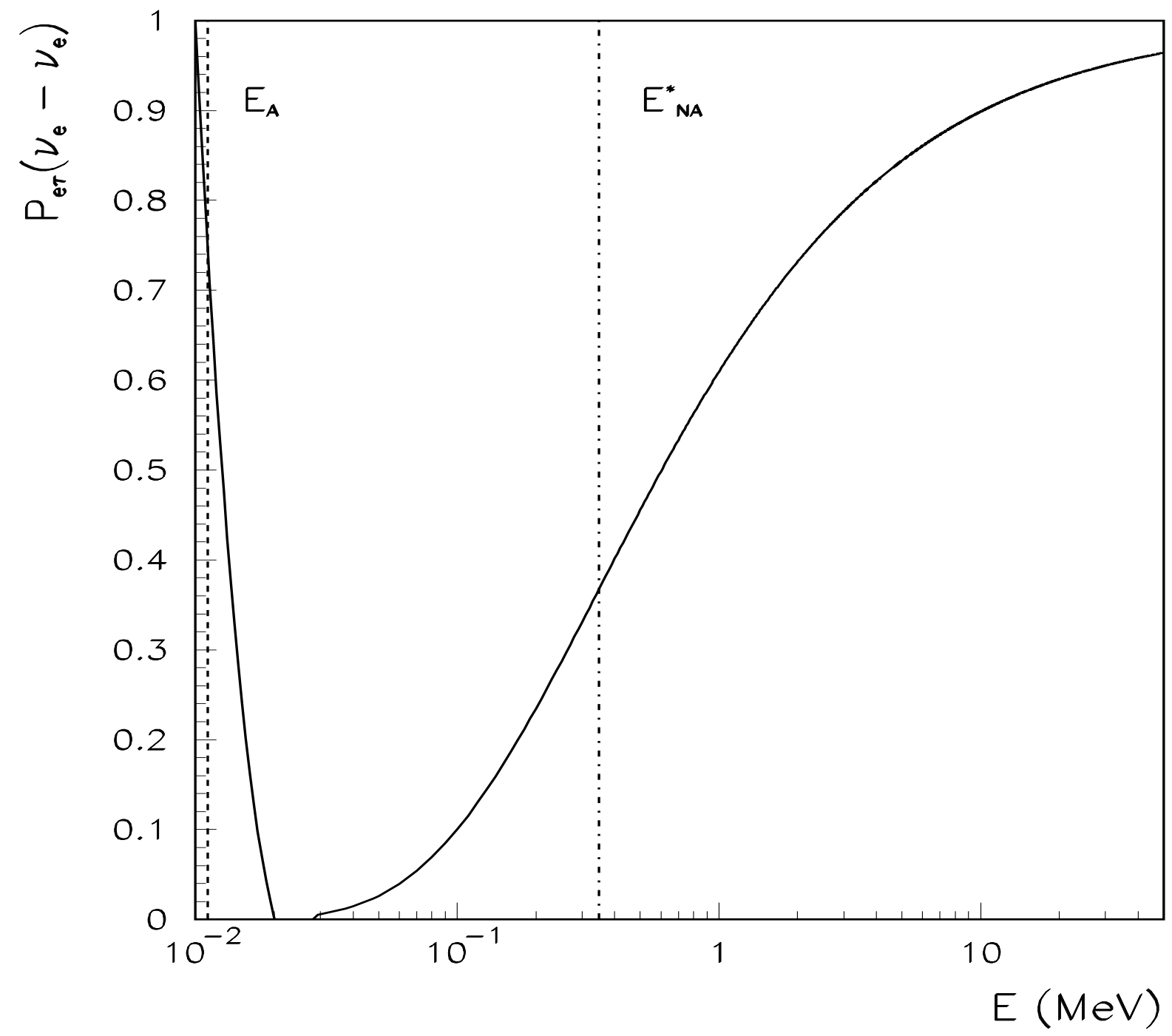

Figure 4: The same quantity of Fig. 3 is shown, but with $\sin ^{2}\left(2 \theta_{e \tau}\right)=3.25 \times 10^{-8}$. 
Fig. 5

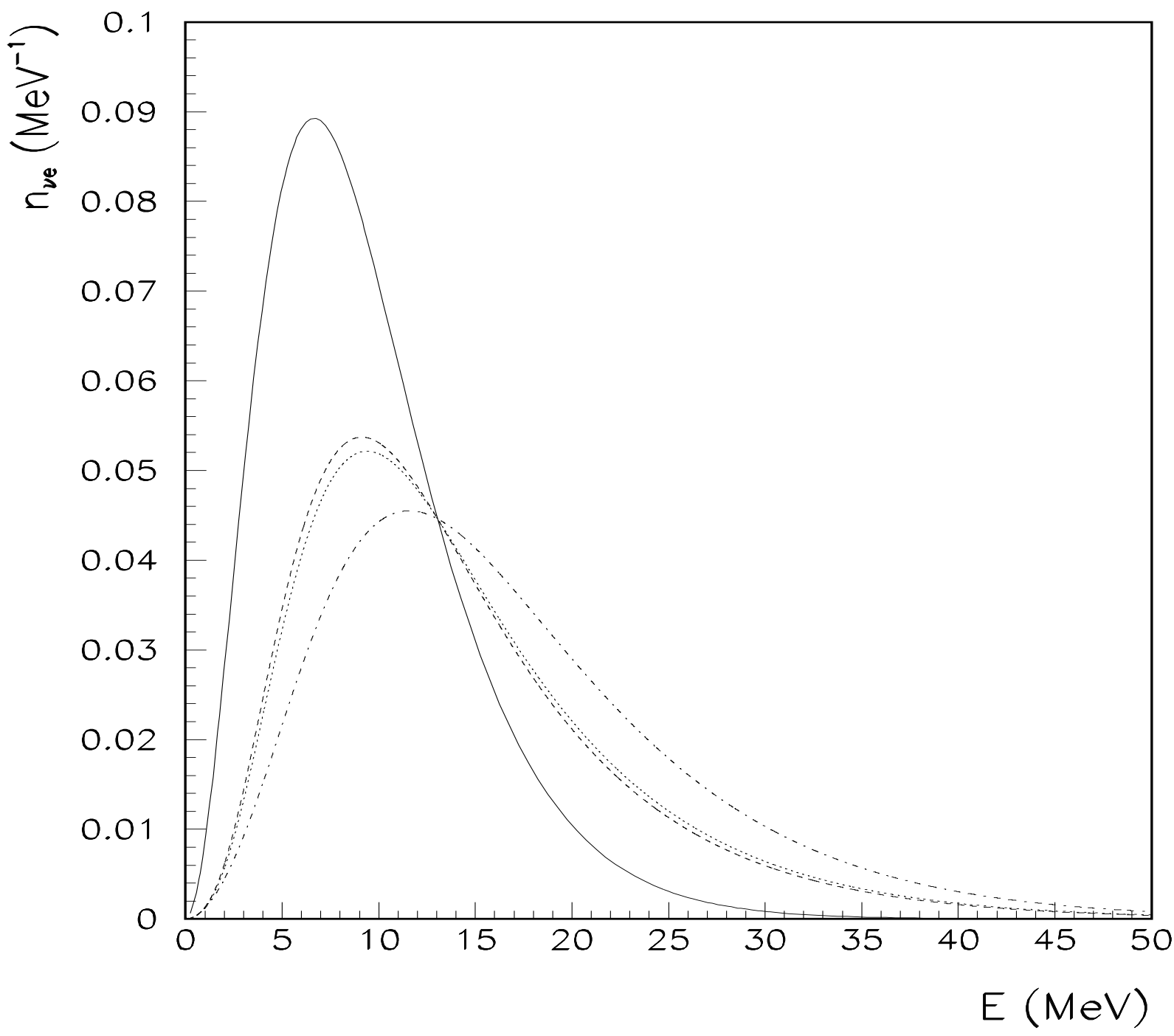

Figure 5: The energy spectra for $\nu_{e}$ are here reported. The solid line represents the pure initial thermal distribution at the neutrinosphere, whilst the dashed and the dotted lines represent the distorted spectrum for $S O(10)$ [2] with $\sin ^{2}\left(2 \theta_{e \tau}\right)=3.25 \times 10^{-10}, 3.25 \times 10^{-8}$, respectively. The dashed-dotted line corresponds to the same quantity for $S U(5)_{S U S Y}$, $S O(10)_{S U S Y}$, in the DHR scheme [6]. 
Fig. 6

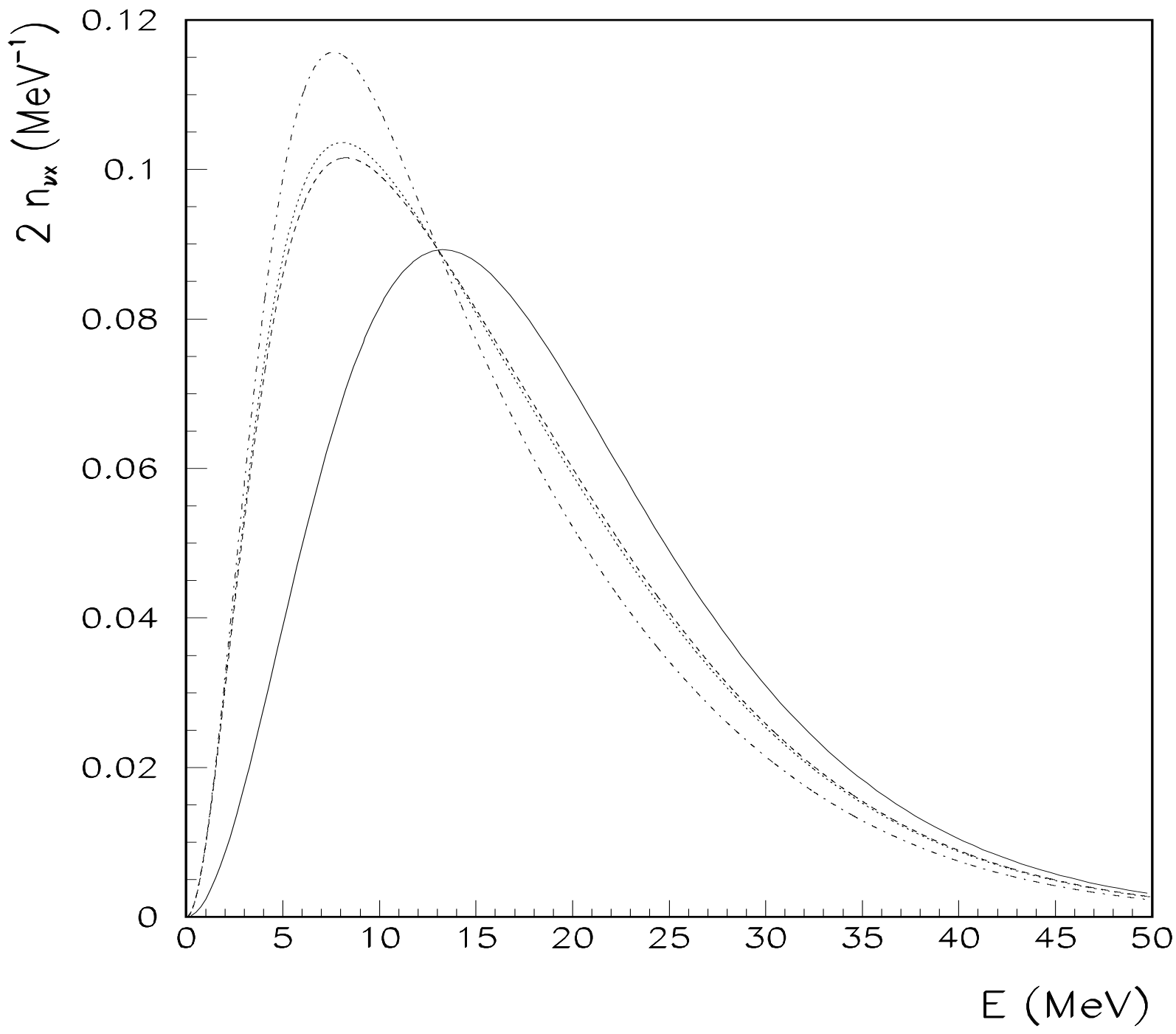

Figure 6: The same quantities of Fig. 5 are here shown, but in this case corresponding to $\nu_{x}$ 